Editorial

\title{
Resistance to hepatitis c virus infection: an overview
}

\author{
Volume 5 Issue 6 - 2017
}

\section{Editorial}

Hepatitis C virus (HCV) infection is a major global health problem. Estimated 180 million people are infected. Spreading of $\mathrm{HCV}$ predominantly is associated with re-use and sharing of injecting apparatus among intravenous drug users (IDUs). Blood transfusion (before the global introduction of serological screening in the early 1990s) and tattooing can cause HCV transmission. About $25 \%$ of persons with acute HCV infection are usually clear the infection. For viral propagation, $\mathrm{HCV}$ depends markedly upon host factors due to encoding very few proteins. HCV particles are identified in complex with very low-density lipoprotein (VLDL) which plays a significant role in transporting triglyceride and cholesterol from the liver to peripheral tissues. Analysis of HCV either isolated from affinity purified from the culture medium of virus-infected Huh7 cells or from patient serum by the electron microscopy (EM) demonstrates structures that contain lipid-rich cores resembling lipoprotein particles rather than the classic viral capsid structure. These findings indicate that the HCV capsid and genome may be hidden within the hydrophobic core of VLDL. There are several receptors for HCV cell entry that based on their interaction with E2, scavenger receptor-B1 and CD81. Occluding and claudin-1, two protein-forming tight junctions also play a role in $\mathrm{HCV}$ cell entry. These receptors function at the later stages of viral cell entry whereas the initial cell binding is at least partially mediated by low-density lipoprotein receptor (LDLR). LDLR does not directly interact with HCV proteins. This cell entry depends on the interaction between the receptor and apoE on the viral particles. Approximately $0.2 \%$ of the world populations have mutations disrupting the function of the LDLR with elevation of the plasma levels of the low-density lipoprotein cholesterol. These affected people can develop premature coronary atherosclerosis. These mutations may protect these persons from infection with $\mathrm{HCV}$ or $\mathrm{HCV}$-related viruses. Additionally, Niemann-Pick C1-liked 1 cholesterol absorption receptor, molecule3-grabbing non-integrins, and heparin sulphate, both dendritic cell and liver specific intracellular adhesion significantly involve in $\mathrm{HCV}$ cell attachment and entry. An RNA interference (RNAi) screen that was used in infectious cell culture system by many investigators may uncovered many host cofactors for HCV replication, with phosphatidylinositol 4-kinase-III $\alpha$ (P14K-III $\alpha$ ), the most consistently identified factor. Silencing of P14K-III $\alpha$ results in an aggregation $\mathrm{HCV}$ replication complexes and double-membrane vesicles. Nine cellular genes that regulate viral replication can be identified by another RNAi screen that targeting approximately 4,000 human genes. After HCV infection (primary infection), an innate immune response is identified in the blood and liver, contributes to induction of antiviral proteins, markedly type 1 interferon's $\alpha$ and $\beta$ and thus many antiviral interferon-stimulated genes. Spontaneous HCV clearance then occurs. $H L A-A^{*} 03, \quad H L A-A^{*} 1101, \quad H L A-B * 27, \quad H L A-B^{*} 57$, and $H L A-C w^{*} 0102$ are associated with HCV clearance, whereas HLA class II alleles, such as $H L A-D Q B 1 * 0301$ and $H L A-D R B 1 * 03$ are the most widely reported association with spontaneous HCV clearance. Persons with HCV primary infection were reported to be 12-times more likely to develop chronic HCV infection.

\author{
Attapon Cheepsattayakorn, ${ }^{1,2,3}$ Ruangrong \\ Cheepsattayakorn ${ }^{4}$ \\ 'Editor-in-Chief, Journal of Human Virology and Retrovirology, \\ USA \\ ${ }^{2}$ Oth Zonal Tuberculosis and Chest Disease center, Thailand \\ ${ }^{3}$ Department of Disease Control, Ministry of Public Health, \\ Thailan \\ ${ }^{4}$ Department of Pathology, Faculty of Medicine, Chiang Mai \\ University, Thailand
}

Correspondence: Attapon Cheepsattayakorn, I0th Zonal Tuberculosis and Chest Disease Center, 143 Sridornchai Road Changklan Muang, Chiang Mai 50 I00, Thailand, Tel 6653 I40767, 6653 276364, Fax 6653 140773, 6653 273590, Email attapon1958@gmail.com, attaponche@yahoo.com

Received: July 23, 2017 | Published: July 24, 2017

A previous study demonstrated that only 5 of 9 patients with successful elimination of a previous $\mathrm{HCV}$ infection were able to spontaneously resolve a subsequent $\mathrm{HCV}$ infection. Persons with chronic HCV infection will develop sustained hepatic necroinflammation and fibrosis, unless cured by antiviral. Some persons with highly exposed but seronegative (HESN) phenotype may be infected with a non-hepatocyte cellular tropism or a replication-defective viral variant, contributing generation of cellular immunity without evidence of viremia or seroconversion, called "occult HCV infection". Long-term treatment (more than 12 weeks) with microsomal triglyceride transfer protein (MTP) inhibitors for blocking VLDL secretion, lowering plasma levels of low-density lipoprotein cholesterol, VLDL, and triglyceride, and treatment of $\mathrm{HCV}$ infection can contribute to the fat accumulation in the liver. Nevertheless, MTP inhibitors have an advantage in targeting a host protein rather HCV proteins, so these drugs are less likely to develop drug-resistance problem that caused by $\mathrm{HCV}$ genome mutations. In conclusion, several epidemiological data indicated that despite repeated exposure and very long-lasting high-risk behaviors, a small subset of persons remain apparently uninfected with HCV. Several persons repeatedly clear $\mathrm{HCV}$ infection through both adaptive and innate immune mechanisms. These data indicate genetic plausibility both immunological protection and resistance to HCV. Further investigations on HESN phenotype are needed.

\section{Acknowledgments}

None.

\section{Conflicts of interest}

None. 